\title{
Multiwavelength Observations of the Unusual Soft X-ray Transient ASASSN-16oh
}

\author{
A.F. Rajoelimanana $^{1 *}$, P. A. Charles ${ }^{2,3}$, D. Buckley ${ }^{4}$, P.J. Meintjes ${ }^{1}$ \\ ${ }^{1}$ Department of Physics, University of the Free State, PO Box 339, Bloemfontein 9300, South \\ Africa \\ ${ }^{2}$ School of Physics and Astronomy, University of Southampton, Southampton SO17 1BJ, UK \\ ${ }^{3}$ Astrophysics, Cosmology and Gravity Centre, Department of Astronomy, University of Cape \\ Town, Rondebosch 7701, South Africa \\ ${ }^{4}$ South African Astronomical Observatory, P.O. Box 9, Observatory, 7935, South Africa
}

E-mail: rajoelimananaadufs.ac.za

\begin{abstract}
We present our results from the analysis of optical and X-ray observations of the unusual Xray transient ASASSN-16oh. The optical light curve revealed that at quiescence the source was fainter $(I=20.3)$ and brightened up to $I=15$ during the outburst. The outburst light curve looks remarkably symmetric, with a total duration of $\sim 300$ days. The optical spectra from SALT show a strong emission line of He II $\lambda$ 4686, He I and some weak Balmer lines. The X-ray and near ultraviolet fluxes correlate with the optical brightness. Such behaviour is unusual amongst the known supersoft sources (hereafter SSS), and is difficult to assimilate into the standard model, but whatever the cause, a period of significantly enhanced mass transfer from the donor is required.
\end{abstract}

5th Annual Conference on High Energy Astrophysics in Southern Africa

4-6 October, 2017

University of the Witwatersrand (Wits), South Africa

\footnotetext{
*Speaker.
} 


\section{Introduction}

SSS are a class of X-ray sources characterised by their soft $(k T \sim 10-75 \mathrm{eV}) \mathrm{X}$-rays and their extreme luminosity (up to $10^{38} \mathrm{erg} \mathrm{s}^{-1}$ ), which suggest an effective emitting radius comparable to the size of a typical white dwarf [四]. The soft X-ray transient ASASSN-16oh was discovered on Dec 22016 with the All-Sky Automated Survey for SuperNovae (ASASSN) as a $V=15.7$ transient [व]. ASASSN-16oh is located in the field of the Small Magellanic Cloud (SMC) at equatorial coordinates $(\mathrm{RA}, \mathrm{DEC})=(01: 57: 43.64,-73: 37: 32.5) \mathrm{J} 2000.0$. [回] proposed a United States Naval Observatory (USNO) source, 9 arcsec away, with quiescent optical magnitudes of $B=20.4$, $R=20.1$ as a USNO counterpart, implying an outburst amplitude of $>4$ magnitudes.

X-ray and UV/Optical follow-up observations were performed with the Swift telescope on Dec 15 2016. [B] measured UVOT magnitudes of $u v w 2=14.9 \pm 0.1 \mathrm{mag}, u v m 2=14.9 \pm 0.1 \mathrm{mag}, u v w 1$ $=15.0 \pm 0.1 \mathrm{mag}, u=15.5 \pm 0.1 \mathrm{mag}, b=16.9 \pm 0.1 \mathrm{mag}, v=16.8 \pm 0.1 \mathrm{mag}$. The Swift-XRT $\mathrm{X}$-ray spectrum is well fitted with a low-temperature blackbody $\left(N_{\mathrm{H}}=5.2 \times 10^{21} \mathrm{~cm}^{-2}\right)$ having a temperature $k T=0.08 \mathrm{keV}$ and an observed flux of $5.2 \times 10^{-12} \mathrm{erg} \mathrm{cm}^{-2} \mathrm{~s}^{-1}(0.1-8 \mathrm{keV})$. At the distance of the SMC, this implies a peak X-ray luminosity of $\sim 10^{37} \mathrm{erg} \mathrm{s}^{-1}$ [B]].

Here we report the optical and X-ray observations of an unusual transient supersoft source, ASASSN-16oh, in the SMC. We use archival X-ray and near ultraviolet observations from SwiftXRT and Swift-UVOT, optical data from the OGLE-IV project and spectroscopic observations from the Southern African Large Telescope (SALT). We discuss the nature of the X-ray source based on our results.

\section{X-ray observations}

ASASSN-16oh was observed with the Swift Ultraviolet/Optical Telescope (UVOT; [䧃]) and the Swift-XRT [5] in a series of observations started on Dec 152016 (13 days after its discovery). It followed the source declining in brightness over three months. The Swift-UVOT telescope uses three near ultraviolet filters $(u v w 1, u v m 2, u v w 2)$, three optical filters $(u, b, v)$ and one clear filter (white). We extracted source counts from a circular region of radius 5.0 arcsec, centered on the source position. The background counts were extracted from a source free background region with a radius of 40.0 arcsec.

For the Swift-XRT data, the event lists were cleaned using the task xrtpipeline (distributed by HEASARC within the HEASOft package) and standard quality cuts. The source events in the $0.3-10 \mathrm{keV}$ energy range were selected for the analysis. The source and background counts were extracted from a circle and annulus region centered on the position of ASASSN-16oh, respectively. The resulting light curves were then corrected for vignetting and PSF losses using the task xrtlccorr. Figure $\mathbb{W}$ shows the Swift-XRT $(0.3-10 \mathrm{keV})$ and Swift-UVOT (uvw1, uvm2, uvw2) light curves of the transient, along with the OGLE-IV light curve. The source is at its maximum brightness in X-ray, ultraviolet and optical around Dec 16 2016, and then is declining in brightness over approximately 2 months.

\section{Optical observations}

ASASSN-16oh lies within the field regularly monitored by the OGLE-IV project since 2010 


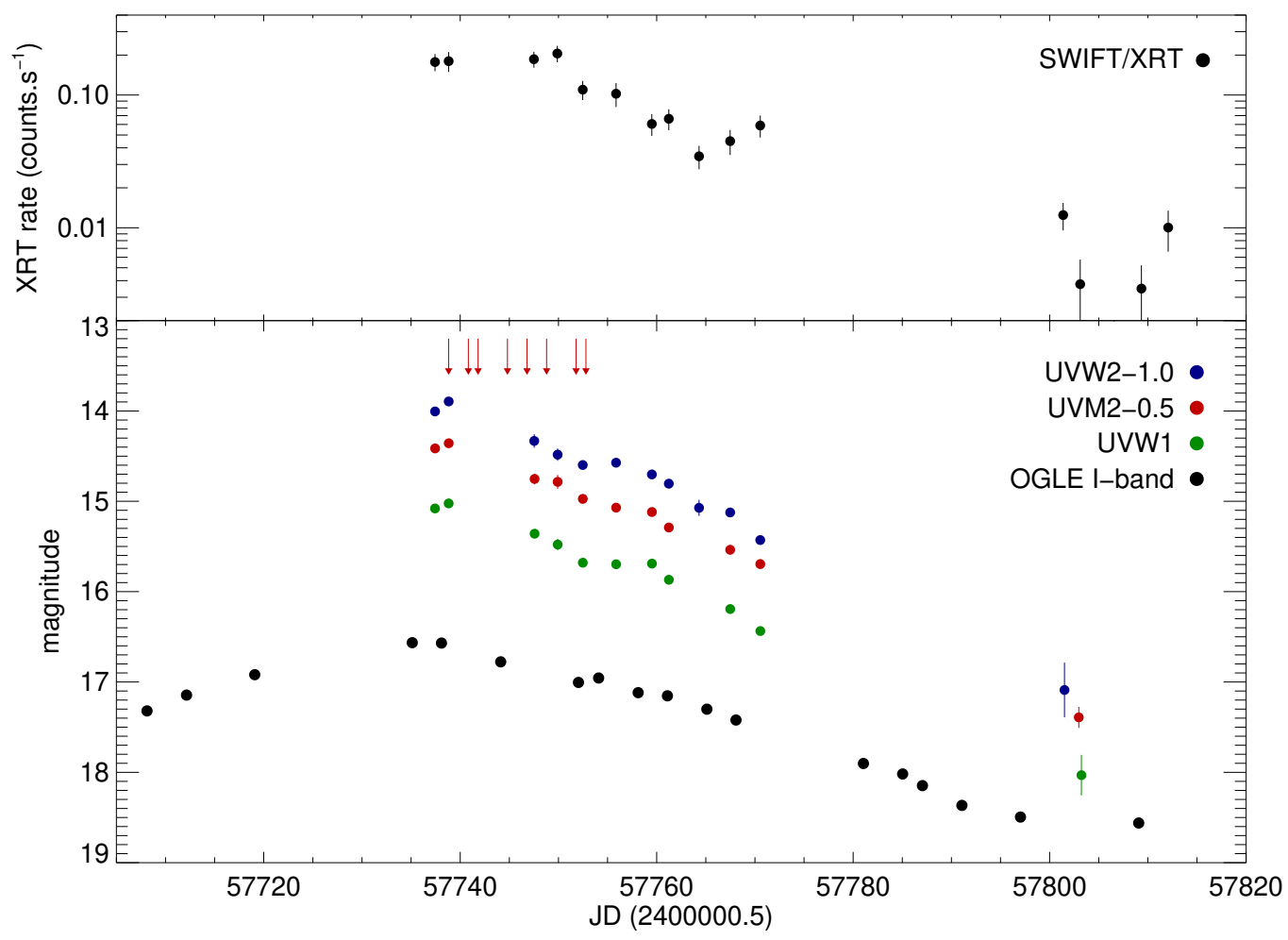

Figure 1: The Swift-XRT 0.3-10 keV light curve (upper panel). Swift-UVOT light curves (blue: UVW2-1.0 mag, red: UVM2-0.5 mag, green: UVW1) and the OGLE-IV I-band light curve (black). The red arrows indicate the times of the SALT optical observations.

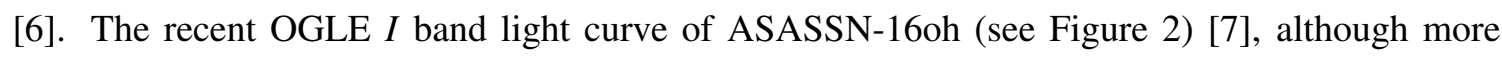
sparsely sampled, shows in detail the optical outburst. The shape of the outburst is quite symmetric with a maximum brightness occurring around Dec 14 2016. The optical brightening is very slow with a rate of 0.02 magnitude per day. Prior to the giant outburst, the source has been an irregular variable for several years, with some weaker outbursts and quiescent flux of $I=20.3$. We did not find any significant period in the OGLE-IV data.

Optical spectroscopy follow-up of ASASSN-16oh were undertaken with SALT [ [ 8 ] between Dec 162016 to Dec 31 2016. Medium resolution spectra were obtained using the Robert Stobie Spectrograph (RSS) [Q, 四] in longslit mode with a 1.5 arcsec slit. We used either the grating PG2300 or the grating PG3000 at an angle of 30.5 and 44.0 degrees, respectively, giving a resolving power of $\sim 2000<R<3800$. The primary reduction of the data from each CCD detector was done with the PYSALT package [U]] which includes overscan, gain and cross-talk correction. We used the standard IRAF software tasks in TWODSPEC for wavelength calibration, sky subtraction and flux calibration. Then, the one-dimensional spectra were extracted using the IRAF task APALL.

Figure [3] shows the average spectrum of ASASSN-16oh obtained with the SALT/RSS, on which all identified main spectral features are marked and labeled. The narrow emission line of He II $\lambda 4686$ completely dominates the optical spectrum. There is also weak variable Balmer (absorption/emission) lines and some He I lines. 


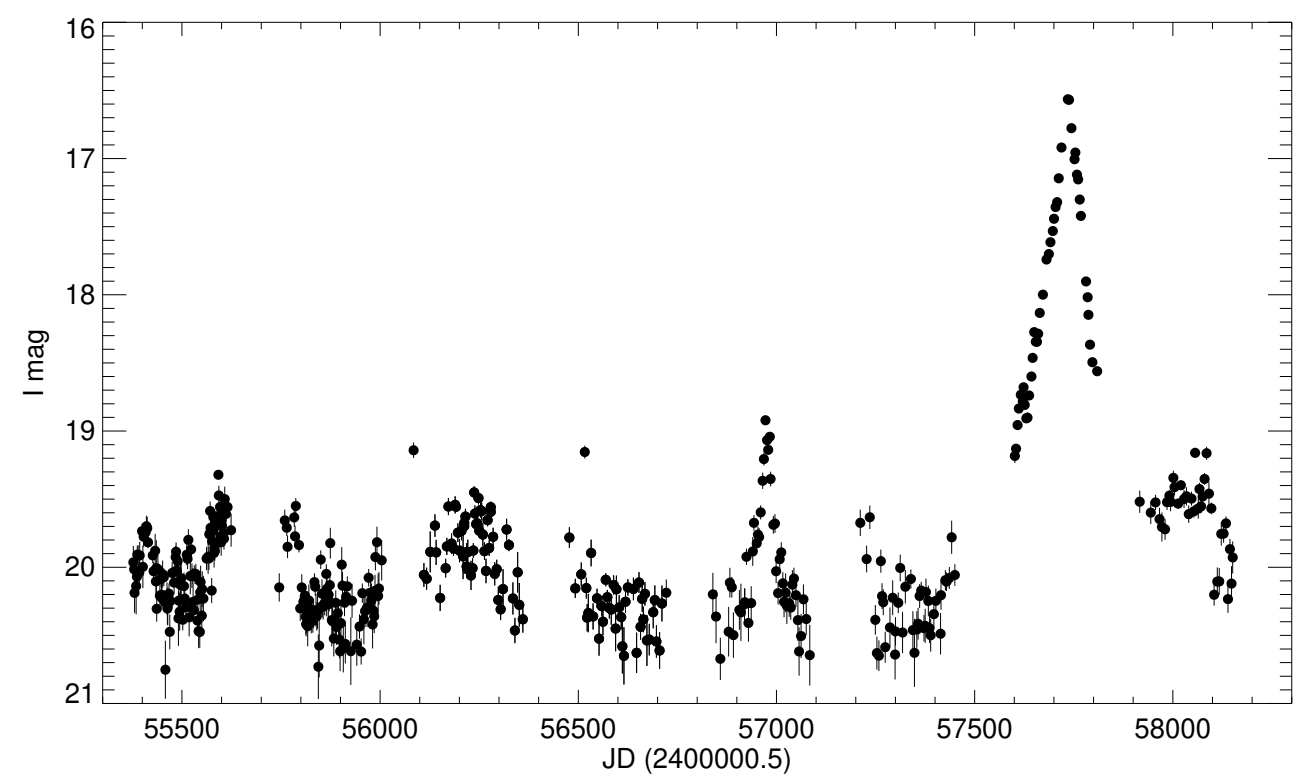

Figure 2: The full OGLE-IV I-band light curve of ASASSN-16oh showing the 3.5 magnitude outburst in Dec 2016.

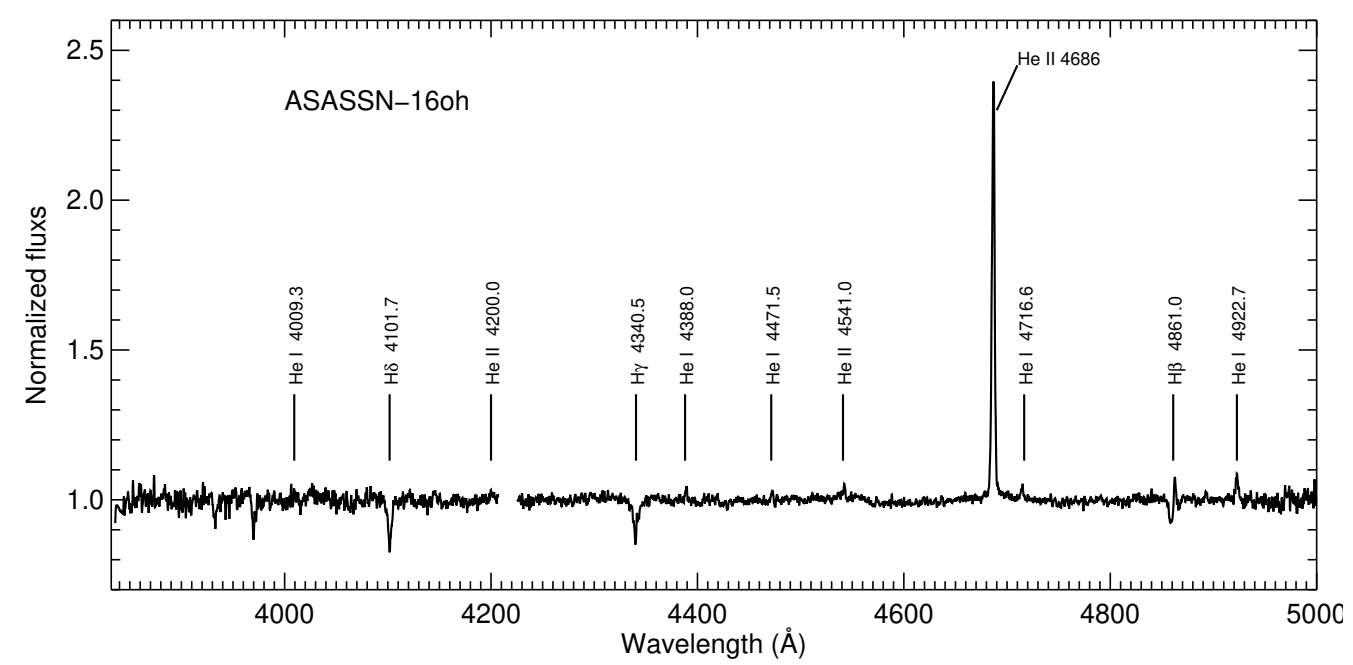

Figure 3: SALT medium-resolution spectrum of ASASSN-16oh in which identified lines are marked.

We measured the radial velocities of the He II $\lambda 4686$ lines by cross-correlating each individual spectrum with the spectrum which has the highest $\mathrm{S} / \mathrm{N}$. We then run a Lomb-Scargle periodogram on the resulting radial velocity dataset. The power spectrum shows its strongest peak at 0.1925 days and evidence of a supra-day period at 5.6619 days. The radial velocity curves folded on both periods are shown in figure $\mathbb{\theta}$. The amplitude of the radial velocity curve is about $35 \mathrm{~km} \mathrm{~s}^{-1}$. 


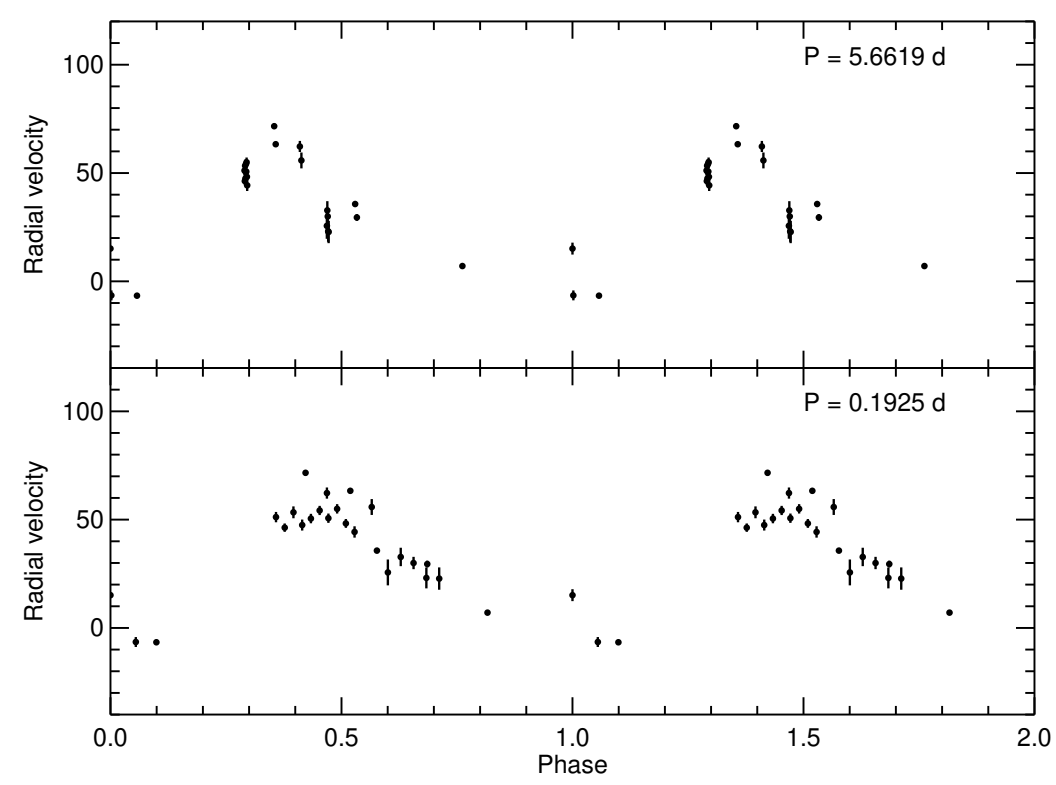

Figure 4: Radial velocity curves of the He II $\lambda 4686$ line folded on the 5.6619 days (top) and 0.1925 days (bottom) for ASASSN-16oh.

\section{Discussion and Conclusion}

During the 2016 outburst, a blackbody fit to the X-ray spectrum gives an effective temperature of $\sim 80 \mathrm{eV}(\sim 900000 \mathrm{~K})$ and an unabsorbed luminosity of about $10^{37} \mathrm{erg} \mathrm{s}^{-1}$ at the distance of the SMC. This gives an emitting radius of $1.4 \times 10^{8} \mathrm{~cm}\left(\sim 0.002 \mathrm{R}_{\odot}\right)$.

For ASASSN-16oh, the supersoft X-ray-on state coincided with the optical and ultraviolet brightening, which is the opposite to that seen in 'classic' supersoft X-ray sources such as CAL83 and RXJ 0513.9-6951, in which the X-ray light curve is anti-correlated with the optical behaviour [[2]. Moreover, the rise-time to the outburst is very slow compared to that seen in 'classic' SSS which would suggest an unusual, gradual entry into the SSS phase.

Two possible mechanisms could explain the X-ray emission. The first one is that the source is a steady nuclear burner and this was triggered by a dwarf nova type outburst. In dwarf nova outbursts (DNO), an instability in the accretion disk, when gas reaches a critical temperature, causes a change in viscosity, resulting in a temporary increase in mass flow through the disc. This heats the whole disc and hence increases its luminosity. The optical outburst seen in ASASSN-16oh has a similar range of amplitude ( $\sim 5 \mathrm{mag}$ ) as in DNO. However, in DNO the source reaches its maximum brightness for only 1-2 day (very fast) but here the source takes $8-9$ months to reach its maximum so a DNO is not possible. Alternatively, If the source isn't nuclear burning, then the X-ray emission would have to be accretion-powered. However, that would require a massive white dwarf (close to the Chandrasekhar Limit) and a very high mass accretion rate.

In the periodogram of the spectroscopic data, the 0.1925 days peak is stronger than the 5.6619 days orbital period. According to [14]], if we assume a $1.3 \mathrm{M}_{\odot}$, we would expect an accretion rate lower limit for stable burning of $3 \times 10^{-7} \mathrm{M}_{\odot} \mathrm{y}^{-1}$. Using the equation (21) from [ए5] and taking $\alpha_{\text {hot }}$, $\alpha_{\text {cold }}$ and $f$ at their fiducial value ( $\alpha_{\text {hot }}=0.1, \alpha_{\text {cold }}=0.02$ and $f=0.4$ ), gives a disc outer radius 
estimate of $1.44 \times 10^{11} \mathrm{~cm}($ see [미])

$$
\dot{M}=3 \times 10^{-7} \mathrm{M}_{\odot} \mathrm{yr}^{-1}\left(\frac{\alpha_{\mathrm{hot}}}{0.1}\right)^{1.14}\left(\frac{\alpha_{\mathrm{cold}}}{0.02}\right)^{-1.23} \times\left(\frac{r_{\text {outer }}}{1.44 \times 10^{11} \mathrm{~cm}}\right)^{2.57}\left(\frac{f}{0.4}\right)^{1.43}
$$

Assuming a mass ratio of 0.5 , if the outer disk radius is near the circularization radius at the time of the outburst, we would expect an orbital period in the range of 4-6 days (in favour of the longer 5.6619 days period). However, if, during the outburst, the outer radius of the disk spreads outwards to the Roche lobe radius of the white dwarf then the most probable orbital period is the shorter 0.1925 days period [ए]6].

\section{Acknowledgments}

Some of the observations reported in this paper were obtained with the Southern African Large Telescope (SALT) under program 2016-2-LSP-001 (PI: Buckley). The OGLE project has received funding from the National Science Centre, Poland, grant MAESTRO 2014/14/A/ST9/00121 to Andrzej Udalski. This work made use of data supplied by the UK Swift Science Data Center at the University of Leicester.

\section{References}

[1] van den Heuvel E. P. J., Bhattacharya D., Nomoto K., Rappaport S. A., Accreting white dwarf models for CAL 83, CAL 87 and other ultrasoft X-ray sources in the LMC, A\&A 262 (1992) 97

[2] Jha S. W., Colmenero E. R., Stanek K. Z., et al., ASASSN-16oh: An Unusual Transient in the Vicinity of the SMC, ATel $\mathbf{9 8 5 9}$ (2016)

[3] Maccarone T. J., Brown P., Mukai K., Swift observations of ASSASN-16oh, ATel 9866 (2016)

[4] Roming P. W. A., Kennedy T. E., Mason K. O., et al., The Swift Ultra-Violet/Optical Telescope, SSRv $120(2005) 95$

[5] Burrows D. N., Hill J. E., Nousek J. A., et al., The Swift X-Ray Telescope, SSRv 120 (2005) 165

[6] Udalski A., XROM and RCOM: Two New OGLE-III Real Time Data Analysis Systems, AcA 58 (2008) 187

[7] Mroz P., Udalski A., Wyrzykowski L., Kozlowski S., Poleski R., OGLE-IV Observations of ASASSN-16oh, ATel 9867 (2016)

[8] Buckley D. A. H., Swart G. P., Meiring J. G., Completion and commissioning of the Southern African Large Telescope, SPIE 6267 (2006) $62670 \mathrm{Z}$

[9] Burgh E. B., Nordsieck K. H., Kobulnicky H. A., Williams T. B., O’Donoghue D., Smith M. P., Percival J. W., Prime Focus Imaging Spectrograph for the Southern African Large Telescope: optical design, SPIE 4841 (2003) 1463

[10] Kobulnicky H. A., Nordsieck K. H., Burgh E. B., Smith M. P., Percival J. W., Williams T. B., O'Donoghue D., Prime focus imaging spectrograph for the Southern African large telescope: operational modes, SPIE 4841 (2003) 1634 
[11] Crawford S. M., Still M., Schellart P., et al., PySALT: the SALT science pipeline, SPIE 7737 (2010) 773725

[12] Rajoelimanana A. F., Charles P. A., Meintjes P. J., Odendaal A., Udalski A., Optical and X-ray properties of CAL 83 - I. Quasi-periodic optical and supersoft variability, MNRAS 432 (2013) 2886

[13] Piro A. L., Bildsten L., Spreading of Accreted Material on White Dwarfs, ApJ 610 (2004) 977

[14] Wolf W. M., Bildsten L., Brooks J., Paxton B., Hydrogen Burning on Accreting White Dwarfs: Stability, Recurrent Novae, and the Post-nova Supersoft Phase, ApJ, 777 (2013), 136

[15] Cannizzo J. K., The Accretion Disk Limit Cycle Model: Toward an Understanding of the Long-Term Behavior of SS Cygni, ApJ, 419 (1993), 318

[16] Maccarone T. J. et al., ASASSN-16oh: a supersoft white dwarf binary without nuclear fusion, Nature, (2018), submitted 